\title{
Design of A New Third-Party Payment Platform Based on Block Chain
}

\author{
Yuqi Wang \\ Institute of management, School of Wuhan University of Technology, Wuhan 430070, China \\ m15872417181@163.com
}

Keywords: third-party payment, platform design, block chain, information security.

\begin{abstract}
Under the background of the rapid development of the third-party payment industry, in order to achieve the demand for information security and transaction data privacy, block chain technology is used in this paper to build a new third-party payment platform. The main research contents include: the third-party payment industry development and the status quo of the block chain technology, through the study of the demand analysis of new type of third-party payment platform, from the application framework, hierarchy and function module of new type of third-party payment platform to carry on the design. The results show that the new tripartite payment platform based on block chain technology can effectively maintain the information security of users.
\end{abstract}

\section{Introduction}

Established as a good credit with the appearance of the Internet financial mechanism of the third-party payment platform, according to iresearch ${ }^{[1]}$, the second quarter of 2017, China's third party mobile payment deals reached 27.1 trillion yuan, up $95.4 \%$ year on year, rose $19.5 \%$. The third-party payment industry is growing rapidly, presenting a blowout development state, and its drawbacks are gradually revealed. In terms of relevant data, it is mainly reflected in information security and privacy issues ${ }^{[2,3]}$. Under the current centralized third-party payment system, users can not get effective guarantee of information security, the possibility of trading data has been tampered with, and the platform of transaction costs and maintenance costs are relatively high, therefore, decentralized system is the trend of The Times.

Considered to be the most potential to trigger the fifth round of subversive revolution - block chain core technology, with its decentralized, security, and tamper-resistant features, provides a development road for the trust model between the financial system. Block chain system generally consists of data layer, network layer, consensus layer, layer, layer and application layer composed of contract, using the data block, through the relevant data encryption and asked poke technology, distributed network system, such as data transmission mechanism, a verification mechanism, and all kinds of consensus algorithm and intelligent contracts to achieve ${ }^{[4]}$.

In third-party payment system based on block chain technology, the transaction data cannot be tampered with, the user can track to their own money and information can be kept secret, this will cause much attention and support, playing a big role in promoting the development of chain block

\section{Platform demand analysis}

New third-party payment platform is composed of multiple function module set of comprehensive system, it is mainly used to serve as the credit intermediary, transaction both sides of the security of network transactions, the user's personal information and trading data from illegal access, illegal use and illegal share. Its design should conform to the four principles: safety, stability, modularization and standardization. It should be convenient for management and maintenance, and can provide safe and reliable service for users and administrators and so on more than one role. This paper mainly from the account management, transaction processing and payment and settlement deal with three basic module design, in order to realize the based business platform system function, and 
maintenance of user information security goals are met.

\section{Platform architecture}

\subsection{Application framework}

Due to operational feasibility and cost-benefit considerations, the new third-party payment platform is applied to the alliance chain, as shown in figure 1:

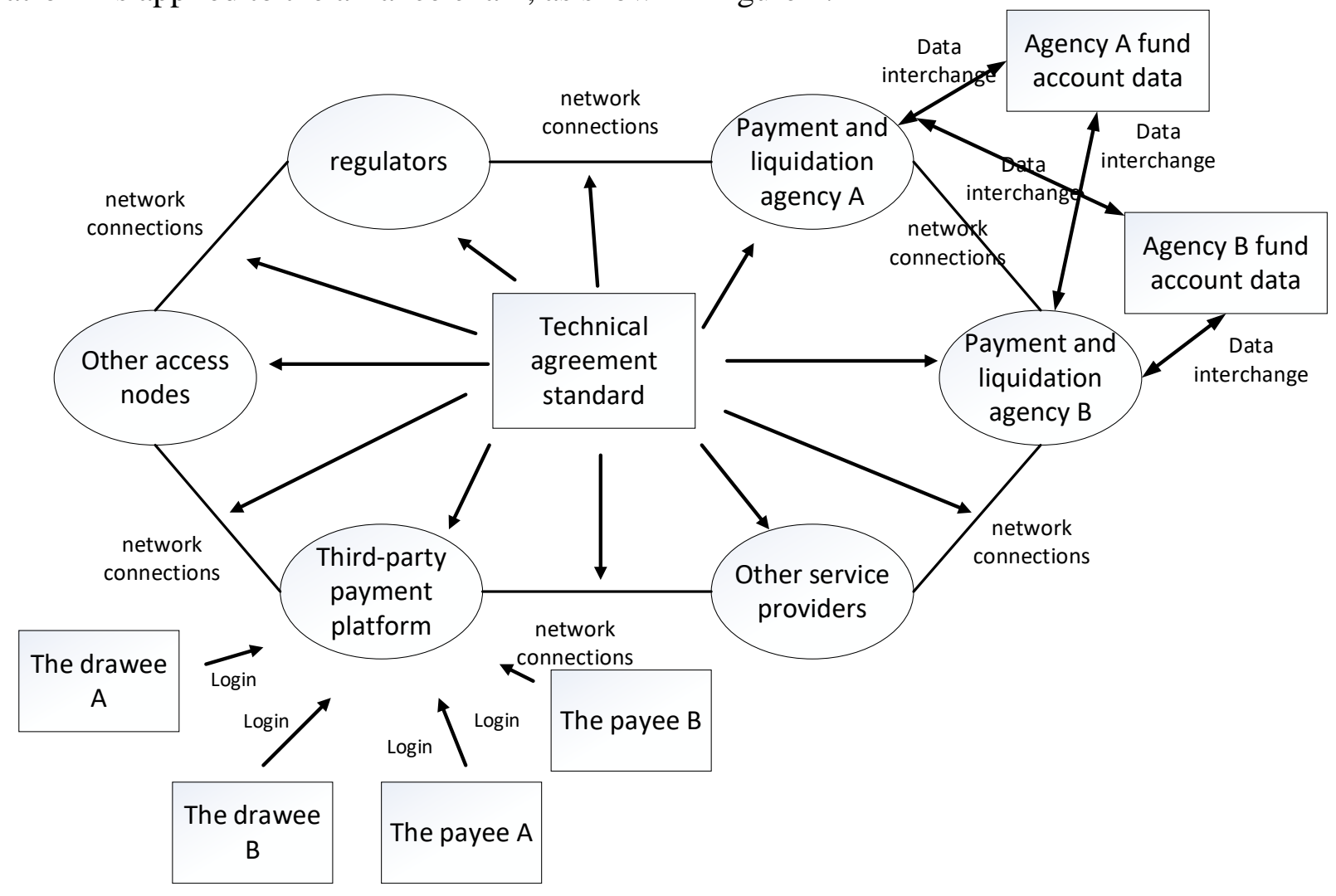

Fig. 1 Application Framework

The technical agreement standard is the key technology of the whole block chain. In a certain sense, the disintermediation of block chain is to remove the human factors in the intermediary, and to provide credibility with strict technical means. Whether the technical agreement standard of public general ledger is accurate, objective and impartial will determine whether the block chain network is effective in market application and whether it can be accepted by participants ${ }^{[5]}$.

The nodes in the alliance chain should include third-party payment platforms, other service supply nodes, payment clearing nodes and regulatory agency nodes. Third-party payment platform and other service supply node is responsible for the legitimate access and management of customer information, transaction information of the agent interaction storage and confidential, in accordance with the agreement standards to set up chain blocks and dock with the other nodes in the network node, is open to individuals involved in the transaction terminal access permissions, and collaborative funds transfer and payment and settlement institutions. Payment and settlement node is responsible for the fund transfer information storage, and transfer funds, and the accuracy of recording cash flow path and upload saved, feedback in time, avoid the illegal misappropriation of funds. Regulator is responsible for the off-site supervision, through the erection block chain network nodes, real-time automatic monitoring the body of the third-party payment platform, information and transaction records and flow of funds, through specific intelligent contract bound irregularities, in accordance with the implementation of the anti-money laundering monitoring. The two sides of the transaction are connected to the node by the client, without the huge data storage and processing pressure to facilitate transactions and avoid moral credit risk ${ }^{[5]}$. It is important to note here that all nodes that join 
the platform are honest nodes.

\subsection{Hierarchical structure}

The new third-party payment platform is divided into three layers. The bottom layer is the block chain layer, which covers the latest block chain technology and forms a decentralized general ledger based on the P2P network. The middle layer is the combination of business logic and block chain, and the local database is used to establish account management, fund management, network payment and other functions. The top layer of the platform provides application services and can complete basic services such as registration, transaction and payment. The hierarchy is shown in figure 2 below.

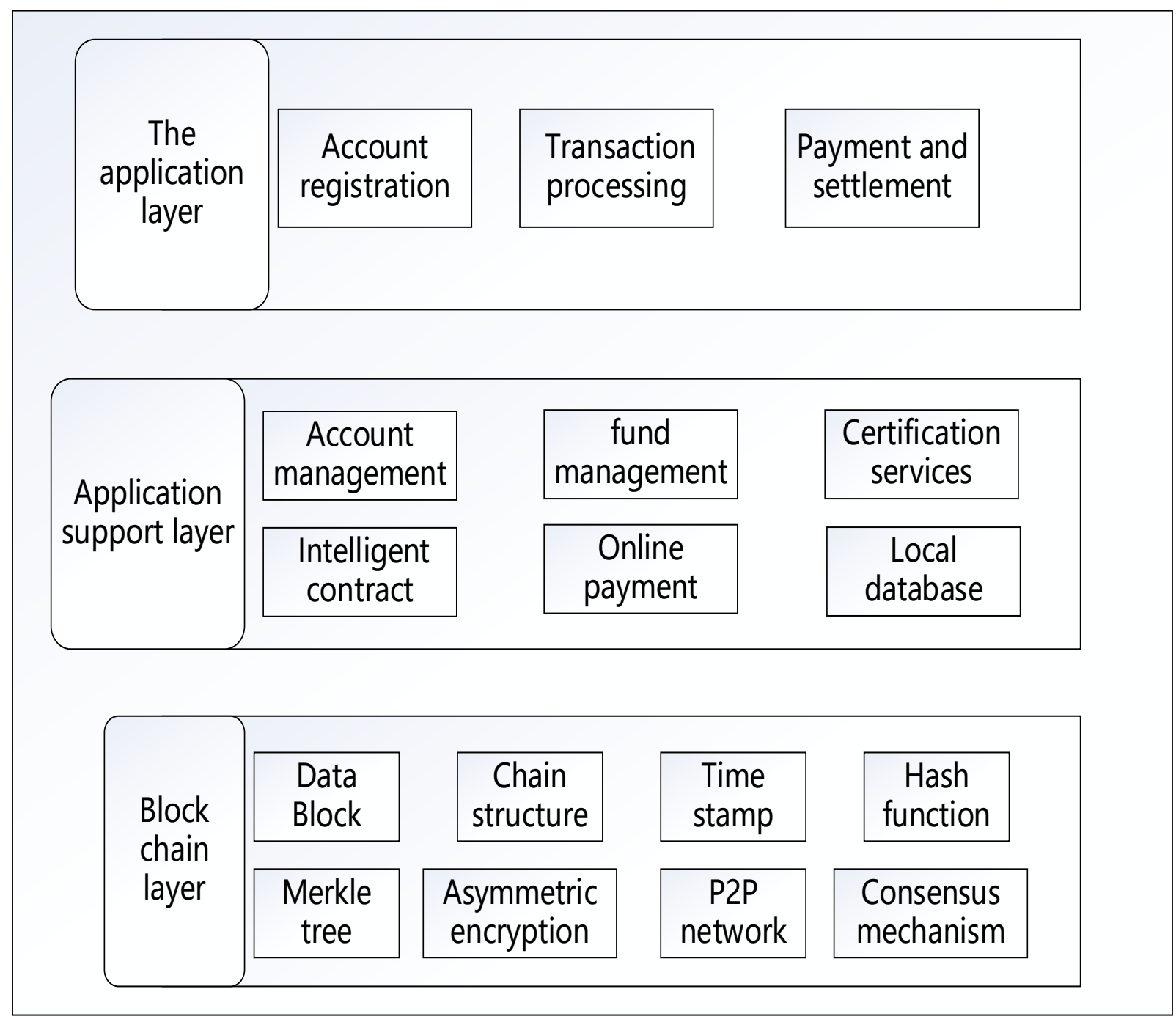

Fig. 2 Hierarchical Structure

\subsection{Function module}

The new third-party payment platform mainly includes the registration, transaction and payment of three business function modules, as shown in figure 3, to realize the customer network transaction. The process is three stages: the first stage is to generate the data blocks, and the information of the client node is encrypted, and then it is put into the newly generated block and broadcast to other nodes. The second stage is the block chain consensus stage, where the successful block is qualified to be included in the block chain. If the verification fails, the error message is returned and the new block is regenerated to continue. The third stage is the data saving stage, where the correct information block is included in the block chain, and the information needed by the client is copied from the block chain to the local database for auxiliary function implementation. 


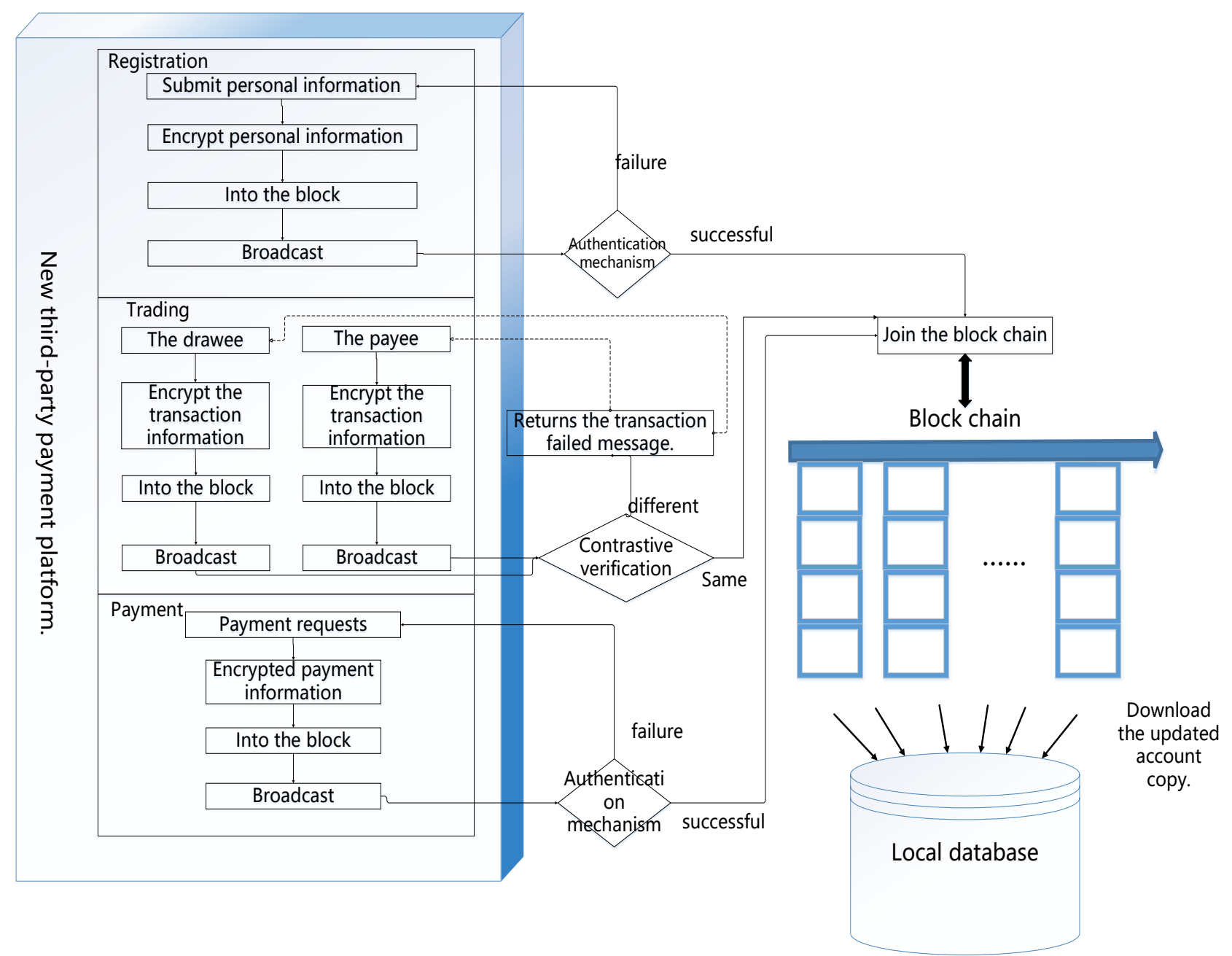

Fig. 3 Functional module diagram

\section{Summary}

This paper analyzes the current status of third-party payment industry and risk, designs a feasible framework and key modules of the new third-party payment platform based on block chain technology, including the safety of the user registration, the privacy of transactions and the confidentiality of payment information. In theory, these functions can be realized. However, due to my limited knowledge and research level, it is simply implemented on the basis of block chain. In future studies, the block chain technology needs to be applied in more fields, such as crowd-funding, smart contracts, and data sharing.

\section{References}

[1] Information on http://www.iresearch.com.cn/report/3076.html.

[2] Jian Sun. Risks and prevention studies of third-party payment platforms in China [J]. Time finance,2017(08):74-75.

[3] Xiao Xiao. Risk type and risk prevention analysis of third-party mobile payment [D]. Southwest university of finance and economics,2016

[4] Yong Yuan, Feiyue Wang. Development status and prospect of block chain technology [J]. Journal of automation,2016,42(04):481-494.

[5] Yu Jian, Wu Weifeng. Exploration of the application of block chain technology in P2P online lending platforms [J]. Tsinghua financial review,2017(06):95-98. 